\title{
Increase in AC-Field Frequency and Recording Performance in Microwave-Assisted Magnetic Recording
}

\author{
R. Koga ${ }^{1}$, F. Akagi ${ }^{1}$, and K. Yoshida ${ }^{2}$ \\ ${ }^{1}$ Graduate School of Electrical Engineering and Electronics, Kogakuin Univ., 1-24-2 Nishisinjuku, \\ Shinjuku-ku, Tokyo, 163-8877 Japan \\ 2 Kogakuin Univ., 1-24-2 Nishisinjuku, Shinjuku-ku, Tokyo, 163-8877 Japan
}

\begin{abstract}
Magnetic recording of a hard disk drive is negatively affected by a trilemma of noise, thermal fluctuation, and writability, which prevents high recording density. Microwave-assisted magnetic recording (MAMR) is a promising recording method for solving this trilemma. In this study, methods for increasing the AC-field frequency generated from a field generation layer (FGL) and recording performance were investigated using a micromagnetic simulator in consideration of magnetic interactions of an MAMR system. Firstly, we discuss methods for increasing the frequency of the AC-field by changing saturation magnetization $\left(M_{s}\right)$ of the FGL. When the $M_{s}$ of the FGL decreased, the AC-field amplitude decreased and its frequency increased. When the $M_{s}$ of the FGL was lower than 1.4 T, the frequency was larger than $20 \mathrm{GHz}$. Secondly, we discuss the recording performances. As a result, to improve recording performance, high frequency of AC-field $(>20 \mathrm{GHz})$ is more important than the strength of STO field.
\end{abstract}

Key words: magnetic recording, microwave-assisted recording, micromagnetic simulation, spin-torque oscillator.

\section{Introduction}

Hard disk drives (HDDs) have been investigated to increase their areal recording density. Recently, however, HDDs have been having simultaneous problems with noise, thermal fluctuation, and writability, commonly known as a trilemma. Microwave-assisted magnetic recording (MAMR) is a promising recording method for solving this trilemma 1-10). In MAMR, a spin torque oscillator (STO) is placed between a conventional write head and a trailing shield. Because the AC-field generated from the field generation layer (FGL) in the STO decreases the switching magnetic fields of the medium, writability can be improved. As a result, the trilemma can be solved. Toshiba demonstrated MAMR read/write system by simulation in 2013 and experimentally demonstrated the write resolution improvement by magnetization flipped type STO in 20149,10). HGST experimentally demonstrated MAMR gain and read/write performance in 2015 ${ }^{11}$. Generally speaking, high frequency and large amplitude for the AC-field are necessary to improve writability. However, performances of STO placed in the write head were poorly understood. The previous paper discussed the STO thickness and magnetic properties for obtaining the strong AC-field. However the frequency was less than $15 \mathrm{GHz}^{12,13)}$.

In this study, we examined methods to increase the frequency of the AC-field and recording performance using a micromagnetic simulator that takes into account magnetic interactions between the write head, medium, and STO. As a result, when the $M_{s}$ of the FGL was lower than $1.4 \mathrm{~T}$, the frequency was larger than 20 GHz. To improve recording performance, high frequency of AC-field (> $20 \mathrm{GHz}$ ) is more important than the strength of STO field.

\section{Calculation model}

Fig. 1 shows the calculation model. The write head consists of a single-pole-type (SPT) head, including a main pole (MP), return pole (RP), and a trailing shield (TS). The medium consists of a soft recording layer, hard recording layer, nonmagnetic layer, and a soft under layer (SUL) and is called an exchange coupled composite (ECC) medium. The STO consists of an FGL and a spin injection layer (SIL). The head and medium was divided into 10-nm rectangular prism cells. A cell of the medium was assumed to be one grain. The STO was divided into 2.5-nm rectangular prism cells. Magnetization dynamic behavior was then calculated using a modified Landau-Lifshitz-Gilbert (LLG) equation with spin torque field $\left(H_{s t}\right)$, as shown below6).

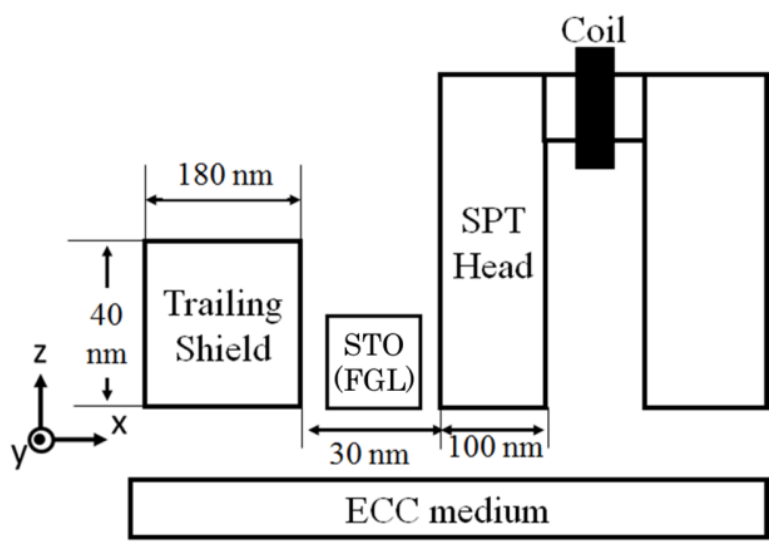

Fig. 1. Calculation model.

$$
\begin{array}{r}
\left(1+\alpha^{2}\right) \frac{d \boldsymbol{M}}{d t}=-\gamma \boldsymbol{M} \times\left(\boldsymbol{H}_{e f f}-\alpha \boldsymbol{H}_{s t}\right) \\
-\frac{\gamma}{M_{S}} \boldsymbol{M} \times\left\{\boldsymbol{M} \times\left(\alpha \boldsymbol{H}_{e f f}+\boldsymbol{H}_{s t}\right)\right\}
\end{array}
$$


Here, $\boldsymbol{M}$ is the magnetization vector, $\boldsymbol{H}_{\text {eff }}$ is the effective field vector, $\alpha$ is the damping constant, $Y$ is the gyro magnetic constant, and $M_{S}$ is the saturation magnetization. The SIL was excluded from the calculation model in this study. However, the $H_{s t}$ injected from the SIL to FGL was calculated using the following equation.

$$
\boldsymbol{H}_{s t}=a_{j} \boldsymbol{M}_{p}=\frac{\hbar \eta J}{2 e M_{s F} d} \boldsymbol{M}_{p}
$$

where $a_{j}$ is the magnitude of the spin torque field vector, $M_{p}$ is the direction vector of $H_{s t}, \hbar$ is Planck's constant divided by 2 п, $\eta$ is the spin polarizability $(0.5$ in this study), $J$ is the inject current density, $e$ is the elementary charge, $M_{S F}$ is the saturation magnetization of the FGL, and $d$ is the FGL thickness. Tables 1-4 show the dimensions and magnetic characteristics of head, medium, and FGL. The current in the coil was $0.18 \mathrm{~A}$. As shown in Fig. 2, the coil current waveform was trapezoidal with a rise time of $0.2 \mathrm{~ns}$ and a cycle of 2.0 $\mathrm{ns}$, corresponding to a bit length of $20 \mathrm{~nm}$ with the relative velocity between head and medium of $20 \mathrm{~m} / \mathrm{s}$. The spacing between the air bearing surface (ABS) and the medium surface was $5 \mathrm{~nm}$. In this simulator, magnetic interactions between the write head, medium, and STO were taken into account. For instance, the AC-field was generated by the precession of the magnetization of the FGL. Basically, the precession occurs due to the balance between the external field (the head-field and stray field from the medium) and the $H_{s t}$ in the perpendicular direction (x component in Fig. 1). However, in actuality, the FGL is affected not only by the $\mathrm{x}$ component but also by the $\mathrm{y}$ and $\mathrm{z}$ components of the external fields. This simulator takes into account all field components and can accurately simulate the processes. Thermal fluctuation was not considered.

Table 1 Magnetic characteristics of Head.

\begin{tabular}{lll}
\hline \hline Symbol & Quantity & Value \\
\hline$M_{S}$ & Saturation & $2.5 \mathrm{~T}$ \\
& magnetization & \\
$K_{u}$ & Magnetic anisotropy & $3.0 \times 10^{3} \mathrm{~J} / \mathrm{m}^{3}$ \\
$A$ & $\begin{array}{l}\text { Exchange stiffness } \\
\text { constant }\end{array}$ & $1.0 \times 10^{-11} \mathrm{~J} / \mathrm{m}$ \\
&
\end{tabular}

Table 2 Magnetic characteristics of FGL.

\begin{tabular}{lll}
\hline \hline Symbol & Quantity & Value \\
\hline$K_{u}$ & Magnetic anisotropy & $5.0 \times 10^{3} \mathrm{~J} / \mathrm{m}^{3}$ \\
$A / /$ & Exchange stiffness & $2.0 \times 10^{-11} \mathrm{~J} / \mathrm{m}$ \\
& constant & \\
\hline
\end{tabular}

Table 3 Dimensions of head, medium, and FGL.

\begin{tabular}{|c|c|c|c|}
\hline \multirow{3}{*}{$\begin{array}{l} \\
\text { SPT } \\
\text { Head }\end{array}$} & & Quantity & $\begin{array}{l}\text { Value } \\
\text { [nm] }\end{array}$ \\
\hline & \multirow[t]{3}{*}{ MP } & Thickness & 100 \\
\hline & & Width & 70 \\
\hline & & Throat height & 50 \\
\hline & \multirow[t]{3}{*}{ TS } & Thickness & 180 \\
\hline & & Width & 540 \\
\hline & & Height & 100 \\
\hline & \multirow[t]{3}{*}{$\mathrm{RP}$} & Thickness & 220 \\
\hline & & Width & 1000 \\
\hline & & Height & 400 \\
\hline \multirow{4}{*}{\multicolumn{2}{|c|}{$\begin{array}{l}\text { Recording Medium } \\
\text { thickness }\end{array}$}} & Recording layer & 10 \\
\hline & & SUL & 50 \\
\hline & & Nonmagnetic & 5 \\
\hline & & layer & \\
\hline \multirow[t]{3}{*}{ FGL } & & Width (y dir.) & 30 \\
\hline & & Height (z dir.) & 30 \\
\hline & & Thickness (x dir.) & 20 \\
\hline \multicolumn{3}{|c|}{ Spacing between MP and FGL } & 5 \\
\hline \multicolumn{3}{|c|}{ Spacing between TS and FGL } & 5 \\
\hline
\end{tabular}

Table 4 Magnetic characteristics of recording layer.

\begin{tabular}{lll}
\hline \hline Symbol & Quantity & Value \\
\hline$M_{S}$ & Saturation magnetization & $0.8 \mathrm{~T}$ \\
$K_{u}$ & $\begin{array}{l}\text { Magnetic anisotropy of } \\
\text { soft layer }\end{array}$ & $3.0 \times 10^{3} \mathrm{~J} / \mathrm{m}^{3}$ \\
$K_{u}$ & $\begin{array}{l}\text { Magnetic anisotropy of } \\
\text { hard layer }\end{array}$ & $1.3 \times 10^{6} \mathrm{~J} / \mathrm{m}^{3}$ \\
$A_{/ /}$ & $\begin{array}{l}\text { Exchange stiffness } \\
\text { constant in plane }\end{array}$ & $2.0 \times 10^{-11} \mathrm{~J} / \mathrm{m}$ \\
$A$ & $\begin{array}{l}\text { Exchange stiffness } \\
\text { constant in vertical }\end{array}$ & $1.0 \times 10^{-11} \mathrm{~J} / \mathrm{m}$ \\
\hline
\end{tabular}

Table 5 Magnetic characteristics of SUL.

\begin{tabular}{lll}
\hline \hline Symbol & Quantity & Value \\
\hline$M_{S}$ & Saturation magnetization & $1.5 \mathrm{~T}$ \\
$K_{u}$ & Magnetic anisotropy & $3.0 \times 10^{3} \mathrm{~J} / \mathrm{m}^{3}$ \\
$A_{/ /}$ & Exchange stiffness constant & $1.0 \times 10^{-11} \mathrm{~J} / \mathrm{m}$ \\
& in plane & \\
$A$ & Exchange stiffness constant & $1.0 \times 10^{-11} \mathrm{~J} / \mathrm{m}$ \\
& in vertical & \\
\hline
\end{tabular}

\section{Methods for increasing frequency of AC-field}

In this chapter, we discuss methods for increasing the frequency of the AC-field. Fig. 2 shows an example of the averaged AC-field in the cross-track direction $\left(H_{y}\right)$ at $9 \mathrm{~nm}$ under the FGL. The averaged amplitude $\left(<H_{y}>\right)$ and frequency $\left(f_{\text {STO }}\right)$ were evaluated. In this graph, circles from 0.5 to $1.0 \mathrm{~ns}$ and from 1.5 to $2.0 \mathrm{~ns}$ show sampling points to evaluate $\left\langle H_{y}\right\rangle$ and $f_{\text {STO }}$ because the AC-field was disordered at the other times due to ununiformity of the gap field during current reversal. Fig. 3 shows the $M_{s}$ of the FGL dependence of $\left\langle H_{y}\right\rangle$ and $f_{\text {STO }}$ at $H_{S t}$ of $80 \mathrm{kA} / \mathrm{m}$. When $M_{S}$ was less than $1.4 \mathrm{~T}, f_{\text {STO }}$ was larger than $20 \mathrm{GHz}$, and its maximum was 22.5 $\mathrm{GHz}$ at $M_{s}$ of $1.2 \mathrm{~T}$. However, $\left\langle H_{y}\right\rangle$ decreased with decreasing $M_{s}$. When $M_{s}$ set $2.0 \mathrm{~T},\left\langle H_{y}\right\rangle$ was $80 \mathrm{kA} / \mathrm{m}$. However, $\left\langle H_{y}>\right.$ was only $36 \mathrm{kA} / \mathrm{m}$ at $M_{s}$ of $1.2 \mathrm{~T}$. Fig. 4 
shows the $M_{S}$ of the FGL dependence of relative variation $\left(\sigma H_{y} /<H_{y}>\right)$ at the same $H_{s t}$ in Fig. 3. When $M_{s}$ was less than $1.0 \mathrm{~T}, \sigma H_{y} \mid\left\langle H_{y}\right\rangle$ drastically increased. In other words, when $M_{S}$ was less than $1.0 \mathrm{~T}$, the AC-field waveform was disordered. Therefore, the AC-field with the frequency over $20 \mathrm{GHz}$ and low $\sigma H_{y} /\left\langle H_{y}\right\rangle$ was obtained on condition that the $M_{S}$ of the FGL was between 1.2 and $1.4 \mathrm{~T}$. We then investigated the increasing method of $\left\langle H_{y}\right\rangle$ for $M_{S}$ of 1.2 and 1.4 T. Fig. 5 shows the $H_{s t}$ dependence of $\left\langle H_{y}\right\rangle$ and $f$ at the $M_{s}$ of the FGL of 1.2 and 1.4 T. Here, $H_{s t}$ changed from 60 to 80 $\mathrm{kA} / \mathrm{m}$. An $f_{S T O}$ over $20 \mathrm{GHz}$ was obtained when $H_{s t}$ was larger than $65 \mathrm{kA} / \mathrm{m}$. For $M_{S}$ of $1.2 \mathrm{~T},\left\langle H_{y}\right\rangle$ increased from 36 to $50 \mathrm{kA} / \mathrm{m}$ as $H_{s t}$ decreased from 80 to $65 \mathrm{kA} / \mathrm{m}$. For the $M_{s}$ of $1.4 \mathrm{~T},\left\langle H_{y}\right\rangle$ increased from 50 to $60 \mathrm{kA} / \mathrm{m}$ as $H_{s t}$ decreased from 80 to $65 \mathrm{kA} / \mathrm{m}$. However, $\left\langle H_{y}\right\rangle$ was lower than the maximum value of $77 \mathrm{kA} / \mathrm{m}$ in Fig. 3.

\section{Recording Performance}

Okamoto et al. experimentally revealed the relation between switching field and AC-field ${ }^{14)}$. According this paper, the switching field monotonically decreased with increasing the frequency and field strength up to a critical frequency depending on the field strength. However, it was difficult to obtain the AC-field with high frequency and strong field, because both have a strong interaction, as shown in the chapter 3. Then, in this chapter, we discuss the recording performances of several AC-fields investigated in the previous chapter. Table 6 and Fig. 6 show recording models. We simulated a high $\left\langle H_{y}\right\rangle$ model $\left(M_{s}=2.0 \mathrm{~T}, H_{s t}=80\right.$ $\mathrm{kA} / \mathrm{m}:$ model A, $M_{s}=1.8 \mathrm{~T}, H_{s t}=80 \mathrm{kA} / \mathrm{m}:$ model B, and $M_{S}=1.6 \mathrm{~T}, H_{s t}=80 \mathrm{kA} / \mathrm{m}$ : model $\mathrm{C}$ ), high frequency models $\left(M_{S}=1.4 \mathrm{~T}, H_{s t}=80 \mathrm{kA} / \mathrm{m}: \operatorname{model~D}\right.$, and $M_{s}=$ $1.2 \mathrm{~T}, H_{s t}=80 \mathrm{kA} / \mathrm{m}:$ model $\left.\mathrm{E}\right)$, a model with over 20 $\mathrm{GHz}$ and maximum $<H_{y}>$ model

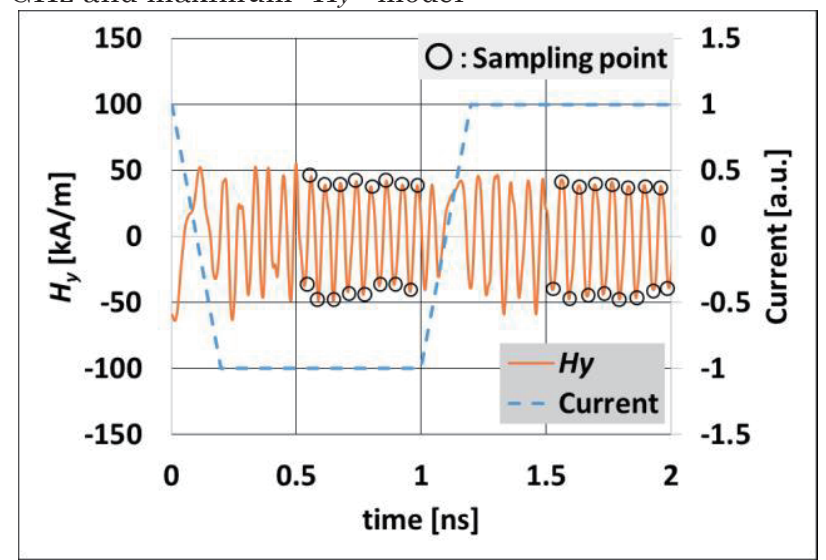

Fig. 2 Example of sampling point of $y^{-}$component of AC-field $\left(H_{y}\right)$ and coil current.

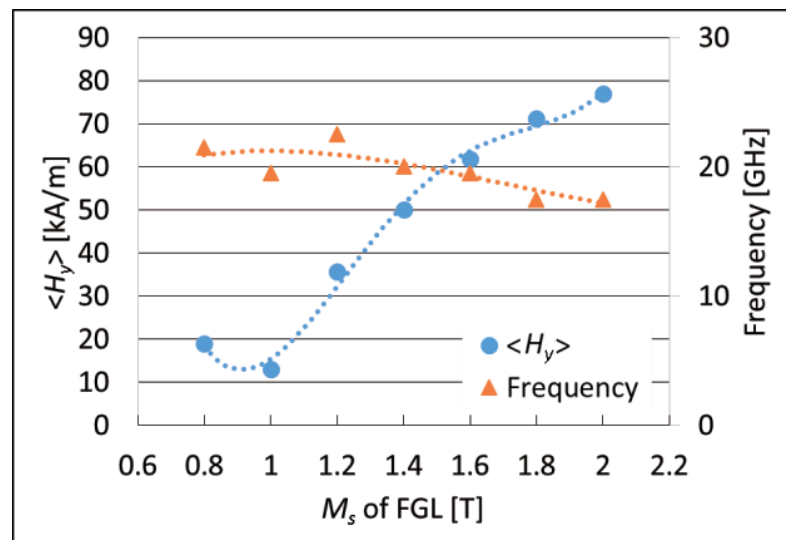

Fig. 3 Averaged y-component of AC-field $\left\langle H_{y}>\right.$ vs. $M_{s}$ of FGL.

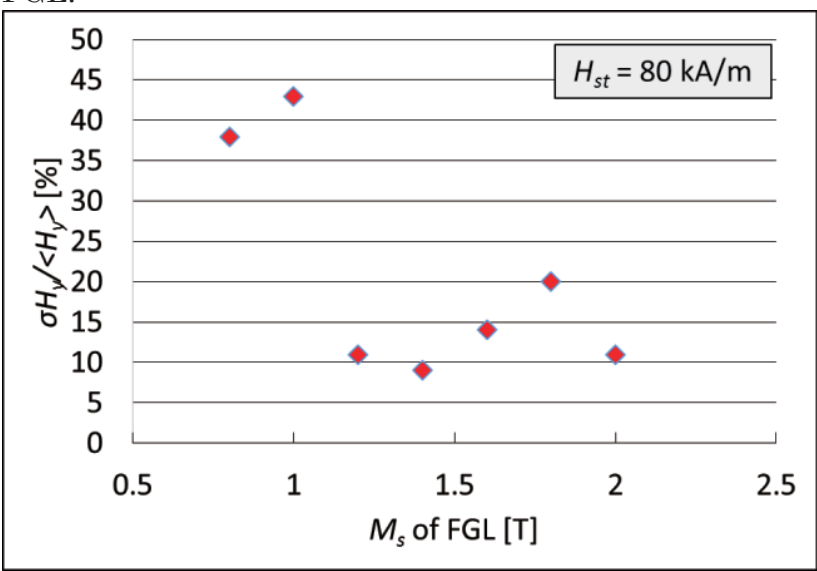

Fig. 4 Normalized variance of averaged $\mathrm{y}^{-}$component of AC-field $\sigma H_{y} /<H_{y}>$ vs. $M_{s}$ of FGL.

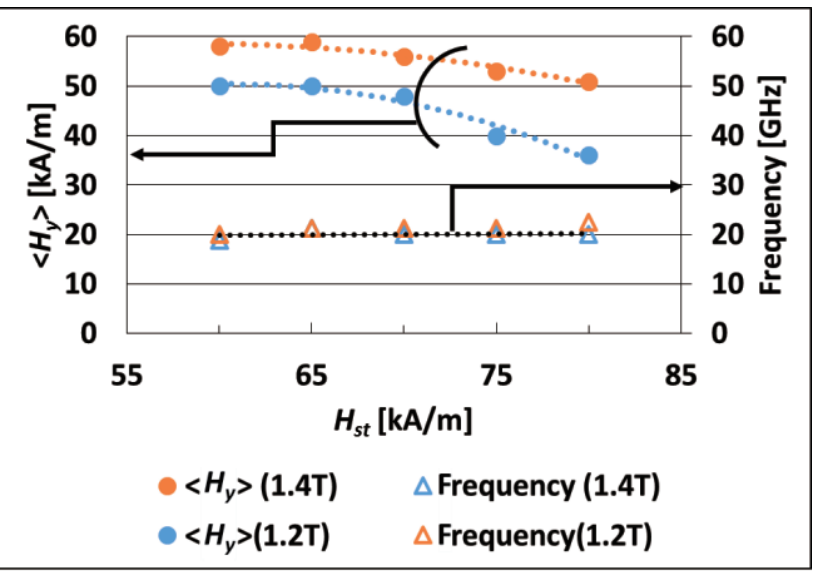

Fig. 5 Averaged y-component of AC-ield $\left\langle H_{y}\right\rangle$ vs. $H_{s t}$.

$\left(M_{s}=1.4 \mathrm{~T}, H_{s t}=65 \mathrm{kA} / \mathrm{m}:\right.$ model $\left.\mathrm{F}\right)$, and a model without an FGL (Model G), which was a conventional write head for comparing the effects of the AC-field. The recording performances were evaluated using recording efficiency (RE), as shown in the following equation.

$$
\mathrm{RE}=\frac{1}{N_{\text {samp }}} \sum_{i=1}^{N_{\text {samp }}} m_{\text {ideal }}(i) \cdot m_{\text {rec }}(i)
$$

Here, $N_{\text {samp }}$ is the sampling number, $m_{\text {ideal }}(i)$ is the $\mathrm{z}$-component of the $i$ th sampling point for the ideal 
magnetization pattern, and $m_{r e c}(i)$ is that for the calculated pattern ${ }^{8}$. The $\mathrm{RE}$ was from -1 to 1 . If the $\mathrm{RE}$ is 1 , all magnetizations of grains are saturated and the medium is ideally recorded. Figs. 7 (a) - (g) show the recorded magnetization waveforms ( $\mathrm{z}^{-}$component) from models $\mathrm{A}$ to $\mathrm{G}$. The ideal waveform is also shown in each figure. The REs from $\mathrm{A}$ to $\mathrm{G}$ were $0.51,0.52$, $0.650 .67,0.83,0.74$, and 0.76, respectively. As a result, the $\mathrm{RE}$ of only model $\mathrm{E}$ was higher than that of conventional write head (model G). On the other hand, the REs of models A-D and F were lower than that of the conventional write head (model G). The $\left\langle H_{y}\right\rangle$ values of these models were higher than that of model E, and the $f_{\text {STOS }}$ were lower than that of model E. Figs. 8 and 9 show $\left\langle H_{y}\right\rangle$ and $f_{S T O}$ dependence of $\mathrm{RE}$, respectively. From these graphs, the REs increased with increasing $f_{\text {STO }}$ and decreasing $\left\langle H_{y}\right\rangle$. Therefore, the model with low $\left\langle H_{y}\right\rangle$ and high $f_{S T O}$ showed a marked improvement in recording performance. As a result, to improve recording performance, high frequency of $\mathrm{AC}$ field ( $>20$ $\mathrm{GHz}$ ) is more important than the strength of STO field.

Table 6 Recording FGL models.

\begin{tabular}{|c|c|c|c|c|c|}
\hline & Model & $\begin{array}{l}M_{S} \\
{[\mathrm{~T}]}\end{array}$ & $\begin{array}{l}H_{s t} \\
{[\mathrm{kA} / \mathrm{m}]}\end{array}$ & $\begin{array}{l}<H_{y}> \\
{[\mathrm{kA} / \mathrm{m}]}\end{array}$ & $\begin{array}{l}f_{S T O} \\
{[\mathrm{GHz}]}\end{array}$ \\
\hline $\mathrm{A}$ & & 2 & 80 & 77 & 17.5 \\
\hline B & High $<H_{y}>$ & 1.8 & 80 & 71 & 17.5 \\
\hline $\mathrm{C}$ & & 1.6 & 80 & 62 & 19.5 \\
\hline $\mathrm{D}$ & High & 1.4 & 80 & 51 & 20.0 \\
\hline $\mathrm{E}$ & frequency & 1.2 & 80 & 36 & 22.5 \\
\hline $\mathrm{F}$ & $\begin{array}{l}f_{S T O} \geqq 20 \mathrm{GHz} \\
<H_{y}>\max \end{array}$ & 1.4 & 65 & 60 & 20.0 \\
\hline
\end{tabular}

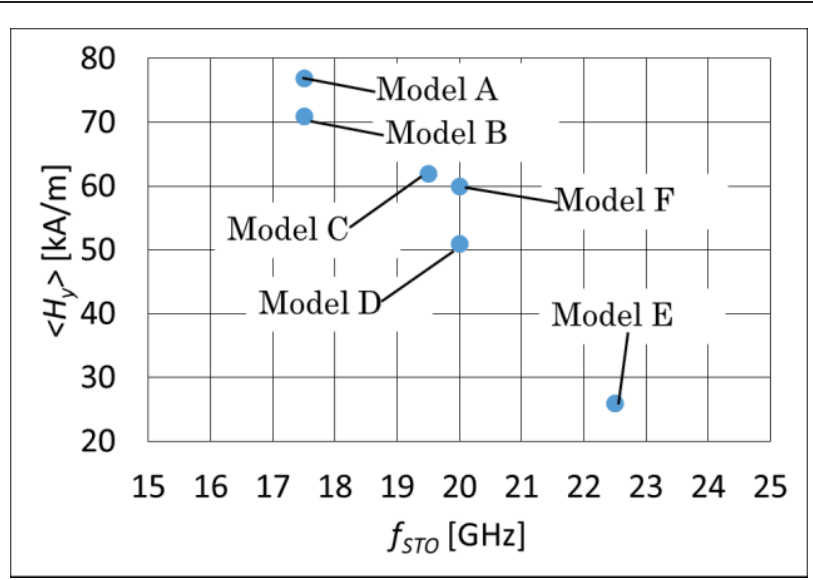

Fig. 6 Relationship diagram of averaged y-component of AC-field $\left\langle H_{y}\right\rangle$ and frequency $f_{\text {STO }}$ of each model.

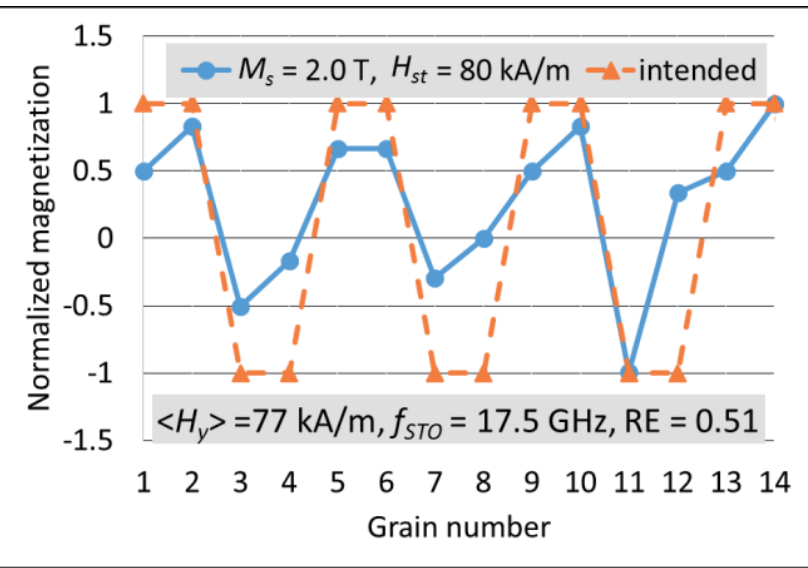

Fig. 7 (a) Recording waveform of model A.

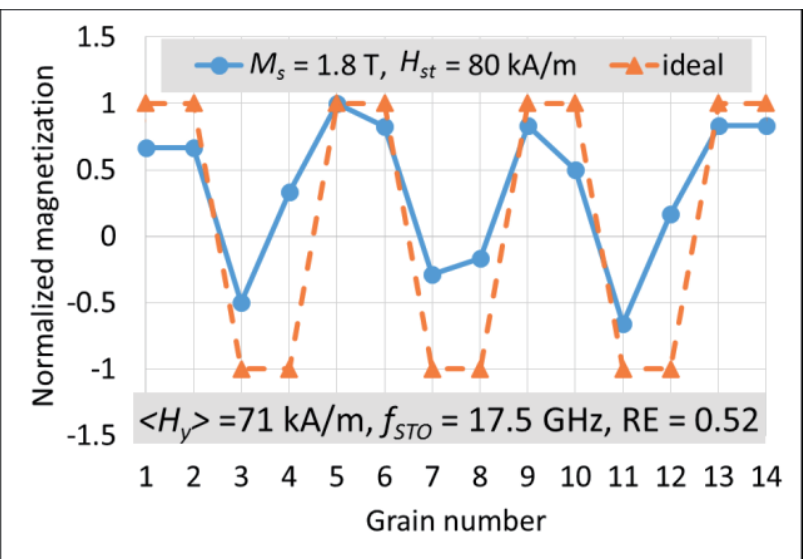

Fig. 7 (b) Recording waveform of model B.

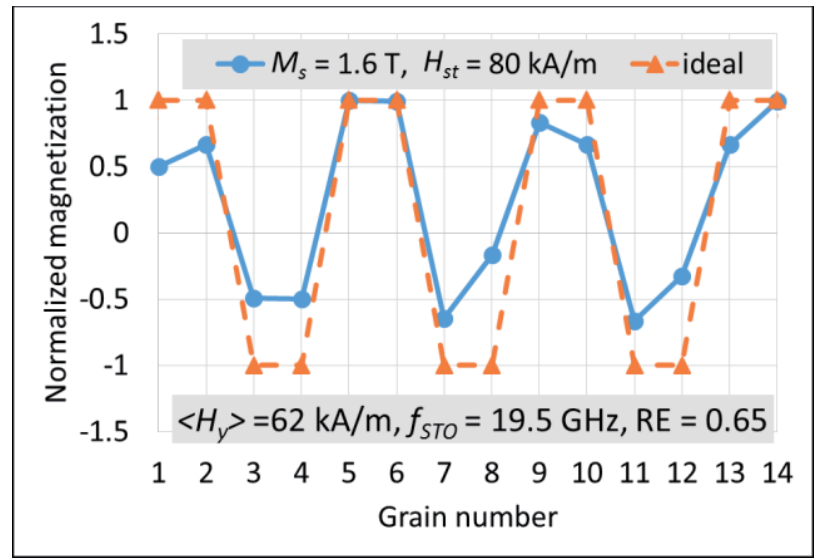

Fig. 7 (c) Recording waveform of model C. 


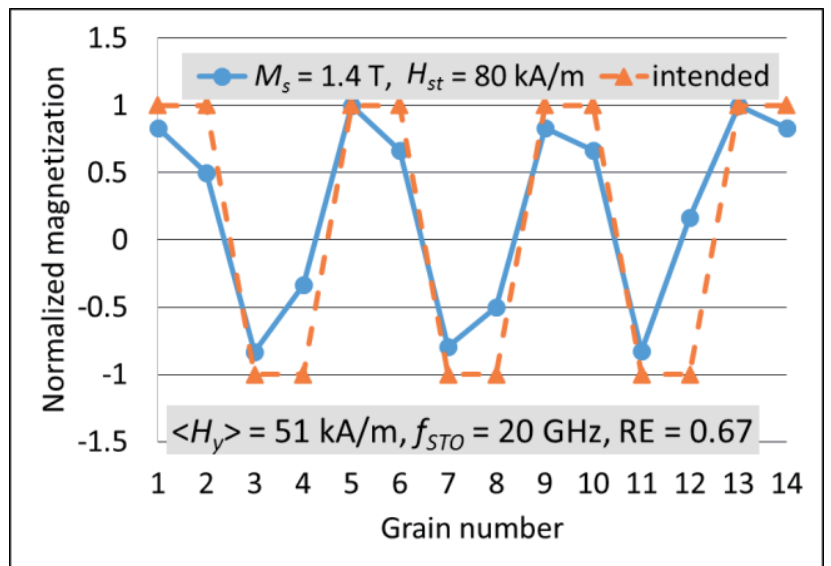

Fig. 7 (d) Recording waveform of model D.

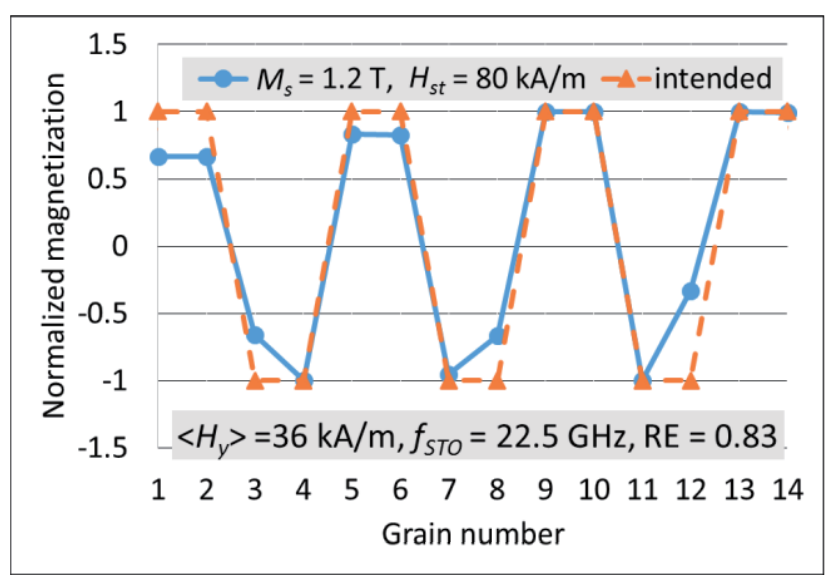

Fig. 7 (e) Recording waveform of model E.

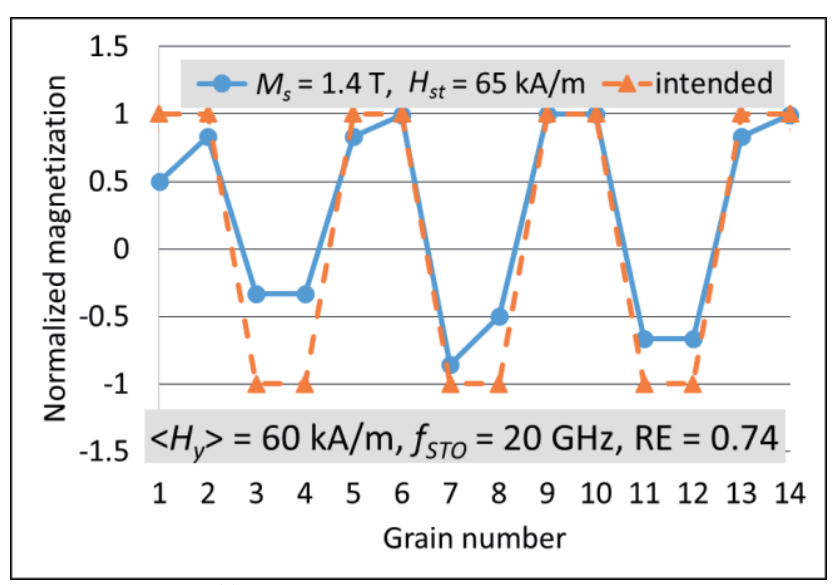

Fig. 7 (f) Recording waveform of model F.

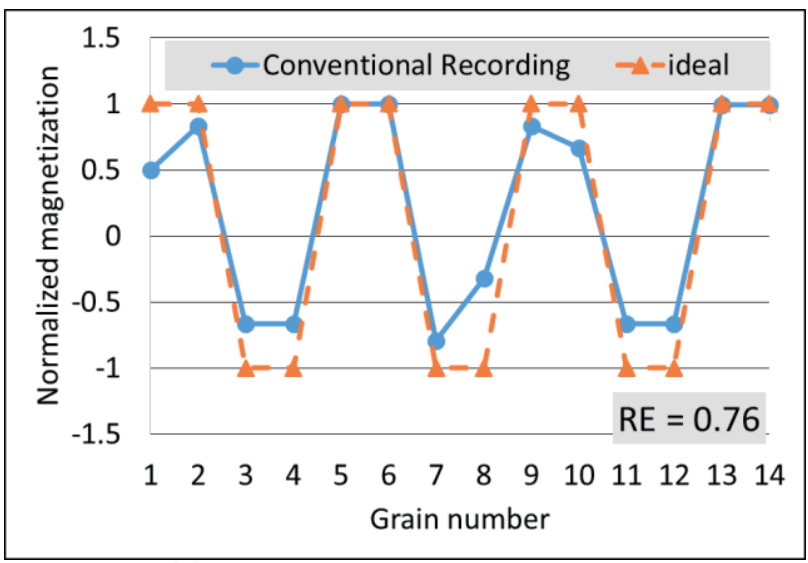

Fig. 7(g) Recording waveform of model G.

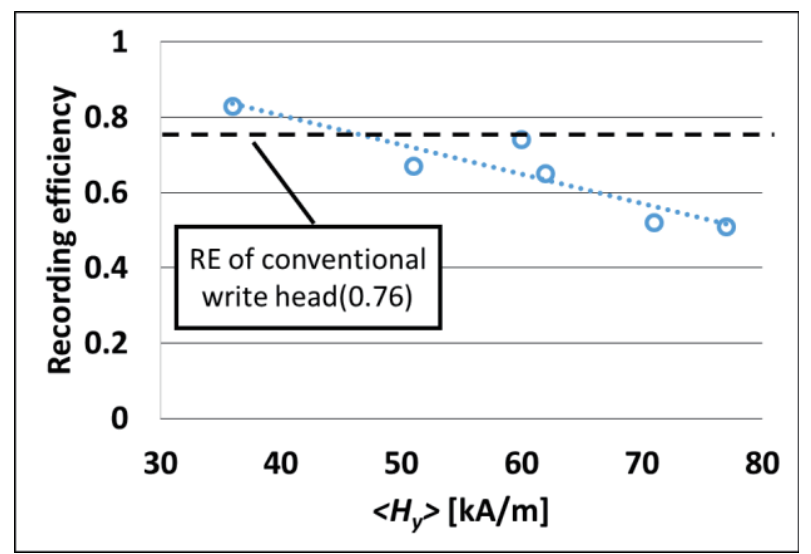

Fig. 8 Averaged y-component of STO field $<H_{y}>$ vs. recording efficiency (RE).

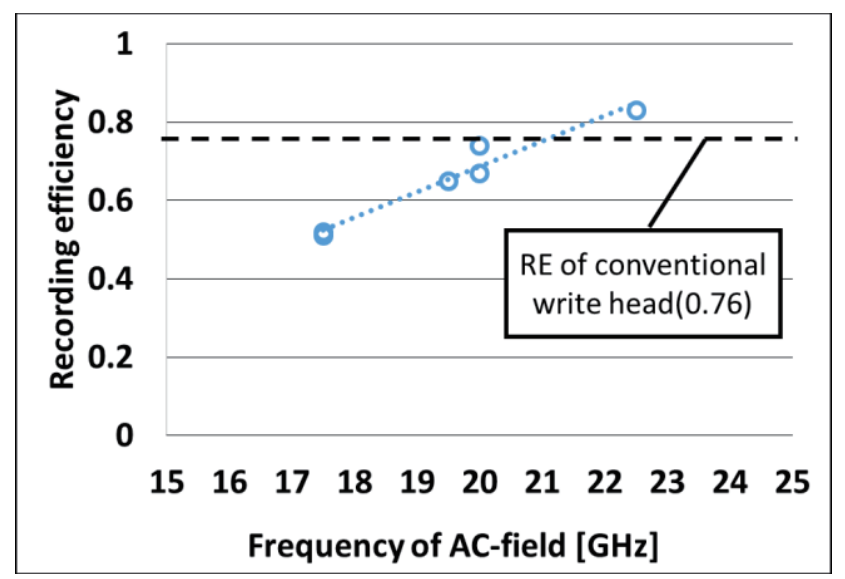

Fig. 9 Frequency of AC-field $f_{\text {STO }}$ vs. recording efficiency (RE). 


\section{Conclusions}

We investigated methods to increase the AC-field frequency and recording performance in microwave-assisted magnetic recording. The following results were obtained.

1. When the $M_{s}$ of the FGL decreases, the AC-field amplitude decreases and its frequency of AC-field increases. When the $M_{S}$ of the FGL is lower than 1.4 T, frequency is larger than $20 \mathrm{GHz}$.

2. To improve recording performance, high frequency of AC-field (> $20 \mathrm{GHz}$ ) is more important than the strength of STO field.

Acknowledgements This study was supported in part by the Storage Research Consortium (SRC), Japan.

\section{References}

1) Y. Tang, and J.-G. Zhu,: IEEE Trans. Magn., 44, 3376 (2008).

2) M. Shiimoto, M. Igarashi, M. Sugiyama, Y. Nishida and I. Tagawa,: IEEE Trans. Magn., 49, 3636, (2013).

3) J-G.Zhu and Y. Wang,: IEEE Trans. Magn., 46, 751, (2010).

4) M. Igarashi, K. Watanabe, Y. Hirayama and Y. Shiroishi, : IEEE Trans. Magn., 48, 3284, (2012).

5) M. Igarashi, Y. Suzuki, Y. Sato, : IEEE Trans. Magn., 46, 3738 (2013).

6) S. Asaka, T. Hashimoto, K. Yoshida and Y. Kanai, : IEICE TRANS. ELECTRON., E96-C, 1484, (2013).

7) T. Takahashi, S. Asaka, K. Yoshida and Y. Kanai, J. Magn. Soc. Jpn., 36, 150 (2012).

8) K. Yoshida,: IEEE Trans. on Magn., 50, 3202504 (2014).

9) K. Yamada, M. Takagishi, K. Koi, A. Takeo,: Dig. TMRC2013 E1 (2013)

10) A. Takeo, G. Koizumi, N. Naita, K. Yamada, W. chen, M. Zhang, Q. Hu, M. Li, and K. Koi: Dig. Intermag2014 AD-2 (2014)

11) I. Tagawa, M. Shiimoto, M. Matsubara, S. Nosaki, J. Aoyama, and Y. Urakami,: Dig. TMRC2015, D6 (2015)

12) R. Koga, F. Akagi, K. Yoshida: IEICE Technical Report, 114, 1 (2014)

13) R. Koga, F. Akagi, K. Yoshida: IEICE TRANS ELECTRON., J98-C, 138 (2015)

14) S. Okamoto, N. Kikuchi, M. Furuta, O. Kitakami, and T. Shimatsu,: Phys. Rev. Lett. 109, 237209 (2012).

Received Aug. 25, 2015; Revised Oct. 30, 2015; Accepted Dec. 04,2015 